\title{
Differential Etiological Profiles and Antibiotic Resistance Patterns of Urinary Tract Infection Among Different Age Categories: A Retrospective Study from a Tertiary General Hospital During a 12-Year Period
}

Lei Huang ( $\sim$ leihuang2031@bjmu.edu.cn )

Peking University First Hospital

\section{Chenwei Huang}

Peking University First Hospital

\section{Yan Yan}

Peking University First Hospital

Liying Sun

Peking University First Hospital

\section{Haixia Li}

Peking University First Hospital

\section{Research}

Keywords: urinary tract infection (UTI), antibiotic resistance, age categories, Escherichia coli, Enterococcus spp.

Posted Date: September 7th, 2021

DOI: https://doi.org/10.21203/rs.3.rs-858819/v1

License: (c) (i) This work is licensed under a Creative Commons Attribution 4.0 International License. Read Full License 


\section{Abstract}

Background: Urinary tract infections (UTIs) are among the most common infections worldwide. With continuing trends of antibiotic resistance, the etiological distribution and antibiotic susceptibility surveillance are of great importance for empirical antimicrobial therapy. However, the data may change with different ages. The aim of this study was to compare the etiological profiles and antibiotic resistance patterns of UTIs sorted by different age categories from a tertiary general hospital during a 12-year period.

Methods: All positive urine culture results from non-repetitive UTI patients in our hospital from January 2009 to December 2020 were collected retrospectively. The microbial distribution and antibiotic resistance rates were analyzed by WHONET 5.6 software. The etiological profiles sorted by different age categories (newborn, pediatric, adult, and geriatric) and antibiotic resistance rates of the top 5 pathogens were analyzed.

Results: A total of 13308 non-repetitive UTI patients were included in our study. Enterococcus faecium was dominant in newborn, and replaced by Escherichia coli in other age categories. The etiological profiles of different age categories were divergent, sorted by genders (male and female) and ward types (outpatient, in-patient, ICU, and emergency). Escherichia coli, Klebsiella pneumoniae, Enterococcus faecalis, Enterococcus faecium, and Pseudomonas aeruginosa were the top 5 pathogens in all age categories. The resistance rates of cefperazone-sulbactam and piperacillintazobactam in Escherichia coli were low in all age categories. The resistance rates of cephalosporins, carbapenems, and fluoroqinolones in Klebsiella pneumoniae were higher in geriatric patients overall. Enterococcus faecium was more resistant than Enterococcus faecalis in all age categories.

Conclusions: The UTI etiological profiles and antibiotic resistance patterns were divergent among different age categories, thus different empirical antibiotic therapies should be considered for different ages.

\section{Introduction}

Urinary tract infections (UTIs) are the infection of urethra (urethritis), bladder (cystitis) or kidney (pyelonephritis). It is among the most common infections worldwide, with substantial morbidity, mortality, and economic burden [1, 2, 3]. Women are more susceptible to UTIs. Over $60 \%$ of women will experience at least one UTI during their lifetime, and $20 \%-30 \%$ of them will experience recurrent UTI within the next 6 months [4]. Additionally, the prevalence of UTI increases with age, which could reach up to $20 \%$ among women older than 65 years [5].

Previous studies showed that uropathogenic Escherichia coli caused approximately $80 \%$ of UTIs [6], while the prevalence of pathogens varied in different regions or different studies $[7,8,9]$. Moreover, the diagnosis and treatment of UTI were different across different age groups [10], e.g. young women with UTI were different from those older than 65 years, and the antibiotic choices for treating UTI were different between pediatric and adult patients. To the best of our knowledge, few studies have systematically compared the UTI etiological profiles and antimicrobial resistance patterns for patients of different ages.

Peking University First Hospital is a 1800-bed tertiary general hospital of Peking University, in Beijing, China. Both nephrology and urology departments are the top 1 key discipline in China, and approximately 14000 urine cultures were ordered annually. Thus the data from large sample size of a 12-year period were retrospectively collected and analyzed to better characterize the etiological profiles and antibiotic resistance patterns among different age categories (newborn, pediatric, adult, and geriatric).

\section{Materials And Methods}




\section{Subjects and study design}

The results of positive urine culture from patients suspected of UTIs, including pathogen identification and antibiotic susceptibility testing, were retrospectively collected from January 2009 to December 2020 in our hospital. Only nonrepetitive isolates (the first isolate from the single patient) were included. A total of 13308 non-repetitive positive urine culture results were collected and analyzed by WHONET 5.6 software. The criteria for a positive urine culture for UTI diagnosis was the pure culture or less than 2 kinds of bacteria grown $\geq 10^{4}$ or $10^{5} \mathrm{cfu} / \mathrm{ml}$, as previously described [11].

The included patients were categorized into 4 different age groups according to WHONET 5.6 software definition [12]: the age $\leq 28$ days was newborn, 28 days $<$ age $\leq 14$ years was pediatric, 14 years $<$ age $\leq 65$ years was adult, and the age $>65$ years was geriatric. The 4 age categories were analyzed and compared for pathogen distribution and their main pathogens' antibiotic susceptibility testing.

\section{Sample collection and culture}

The procedure of urine culture and subculture were performed following the standard operation procedure (SOP) by the Department of Clinical Laboratory in our hospital. Briefly, clean-catch midstream urine was collected from patients suspected of UTIs, then samples were sent to clinical microbiology laboratory within 2 hours. $10 \mu$ urine with calibrated loop was streaked into both Columbia blood agar plate and China blue lactose rosolic acid agar plate, then incubated at $35^{\circ} \mathrm{C}$ aerobically for $24-48$ hours with $5 \% \mathrm{CO}_{2}$ atmosphere. The number of colony was counted and calculated to concentration with unit of $\mathrm{CFU} / \mathrm{ml}$.

\section{Bacteria identification and antibiotic susceptibility testing}

The bacterial identification and antibiotic susceptibility testing were performed by VITEK 2 Compact automated system (bioMeriux, France) or Phoenix 100 automated system (BD, USA), and the Minimal Inhibitory Concentration (MIC) of each antibiotic was determined. The MICs were judged to be susceptible, intermediate, or resistant following the breakpoints of CLSI M100 document.

Escherichia coli ATCC 25922, Pseudomonas aeruginosa ATCC 27853, and Staphylococcus aureus ATCC 25923 strains were used for quality control of antibiotic susceptibility testing.

\section{Statistical analysis}

WHONET 5.6 software was used to analyze the microbial distribution and antibiotic susceptibility rates, and the breakpoint of each antibiotic was referenced from CLSI M100 document 31th edition [13]. Categorical variables were analyzed by chi-square test or Fisher's exact test, and continuous variables were analyzed by the Mann-Whitney $U$ test. $\mathrm{P}<0.05$ was considered statistically significant. GraphPad Prism version 8.0 (GraphPad, San Diego, CA, United States) was used to perform the analyses and draw the figures.

\section{Results}

The positive rate of urine culture was approximately $10 \%$ overall during the 12 -year period. A total of 13308 nonrepetitive UTI patients were included and analyzed. Figure 1 showed the overall etiological profile of different age categories. Enterococcus faecium was dominant in newborn $(45 \%, \mathrm{n}=105)$, and replaced by Escherichia coli in pediatric $(34 \%, n=362)$, adult $(43 \%, n=3416)$, and geriatric $(40 \%, n=1617)$ respectively.

Generally, the etiological profiles of different age categories were divergent, sorted by genders (male and female) and ward types (outpatient, in-patients, ICU, and emergency). The comparison of etiological profile between male and female among different age categories were summarized in Table 1. The etiological profile was more diverse in adult male than 
in other age categories, with 13 kinds of bacteria of portion $>2 \%$. Enterococcus faecalis was dominant in newborn male and female, which accounted for $39 \%$ and $51 \%$ respectively. Escherichia coli became dominant gradually with the increasing age, especially in female, which accounted for $41 \%, 50 \%$, and $50 \%$ in pediatric, adult, and geriatric respectively, while this trend was not obvious in male.

Table 1

Comparison of etiological profile between male and female among different age categories

\begin{tabular}{|c|c|c|c|c|c|c|c|c|}
\hline \multirow[t]{3}{*}{ Organism } & \multicolumn{2}{|c|}{ newborn } & \multicolumn{2}{|c|}{ pediatric } & \multicolumn{2}{|l|}{ adult } & \multicolumn{2}{|c|}{ geriatric } \\
\hline & male & female & male & female & male & female & male & female \\
\hline & $\begin{array}{l}(\mathrm{N}= \\
111)\end{array}$ & $\begin{array}{l}(\mathrm{N}= \\
115)\end{array}$ & $\begin{array}{l}(\mathrm{N}= \\
504)\end{array}$ & $\begin{array}{l}(\mathrm{N}= \\
562)\end{array}$ & $\begin{array}{l}(\mathrm{N}= \\
2208)\end{array}$ & $\begin{array}{l}(n= \\
5438)\end{array}$ & $\begin{array}{l}(\mathrm{N}= \\
1559)\end{array}$ & $\begin{array}{l}(\mathrm{N}= \\
2470)\end{array}$ \\
\hline Escherichia coli & $20 \%$ & $19 \%$ & $25 \%$ & $41 \%$ & $27 \%$ & $50 \%$ & $24 \%$ & $50 \%$ \\
\hline Enterococcus faecalis & $9 \%$ & $4 \%$ & $14 \%$ & $6 \%$ & $11 \%$ & $9 \%$ & $8 \%$ & $4 \%$ \\
\hline $\begin{array}{l}\text { Pseudomonas } \\
\text { aeruginosa }\end{array}$ & $3 \%$ & 0 & $7 \%$ & $4 \%$ & $10 \%$ & $3 \%$ & $11 \%$ & $4 \%$ \\
\hline Enterococcus faecium & $39 \%$ & $51 \%$ & $19 \%$ & $22 \%$ & $9 \%$ & $6 \%$ & $11 \%$ & $9 \%$ \\
\hline Klebsiella pneumoniae & $8 \%$ & $17 \%$ & $9 \%$ & $12 \%$ & $8 \%$ & $8 \%$ & $14 \%$ & $13 \%$ \\
\hline $\begin{array}{l}\text { Staphylococcus } \\
\text { epidermidis }\end{array}$ & $4 \%$ & 0 & 0 & 0 & $3 \%$ & $3 \%$ & $2 \%$ & 0 \\
\hline Burkholderia cepacia & 0 & 0 & 0 & 0 & $3 \%$ & 0 & 0 & 0 \\
\hline $\begin{array}{l}\text { Acinetobacter } \\
\text { baumannii }\end{array}$ & $2 \%$ & 0 & 0 & 0 & $3 \%$ & 0 & $3 \%$ & 0 \\
\hline Proteus mirabilis & 0 & 0 & $3 \%$ & $2 \%$ & $3 \%$ & $4 \%$ & $6 \%$ & $5 \%$ \\
\hline $\begin{array}{l}\text { Staphylococcus } \\
\text { aureus }\end{array}$ & 0 & 0 & 0 & 0 & $3 \%$ & $2 \%$ & $2 \%$ & 0 \\
\hline Enterobacter cloacae & $3 \%$ & 0 & $4 \%$ & 0 & $2 \%$ & 0 & $3 \%$ & 0 \\
\hline Morganella morganii & 0 & 0 & $2 \%$ & 0 & $2 \%$ & 0 & 0 & 0 \\
\hline $\begin{array}{l}\text { Staphylococcus } \\
\text { haemolyticus }\end{array}$ & 0 & 0 & $2 \%$ & 0 & $2 \%$ & 0 & 0 & 0 \\
\hline Klebsiella aerogenes & $3 \%$ & 0 & 0 & 0 & 0 & 0 & 0 & 0 \\
\hline Klebsiella oxytoca & $5 \%$ & 0 & $2 \%$ & 0 & 0 & 0 & 0 & 0 \\
\hline $\begin{array}{l}\text { Enterococcus } \\
\text { gallinarum }\end{array}$ & 0 & $2 \%$ & 0 & $2 \%$ & 0 & 0 & 0 & 0 \\
\hline Serratia marcescens & 0 & $2 \%$ & 0 & 0 & 0 & 0 & 0 & 0 \\
\hline $\begin{array}{l}\text { Streptococcus } \\
\text { agalactiae }\end{array}$ & 0 & 0 & 0 & 0 & 0 & $5 \%$ & 0 & 0 \\
\hline Others $(<2 \%)$ & $4 \%$ & $5 \%$ & $13 \%$ & $11 \%$ & $14 \%$ & $10 \%$ & $16 \%$ & $15 \%$ \\
\hline
\end{tabular}

The comparison of etiological profile among different ward types (outpatient, inpatient, ICU, and emergency) was summarized in Table 2. Due to the small sample size of newborn and pediatric when divided into different ward type 
subgroups, the data of these two categories were not included for analysis.

Table 2

Comparison of etiological profile among different ward types among the age categories of adult and geriatric

\begin{tabular}{|c|c|c|c|c|c|c|c|c|}
\hline \multirow[t]{3}{*}{ Organism } & \multicolumn{4}{|l|}{ adult* } & \multicolumn{4}{|l|}{ geriatric* } \\
\hline & outpatient & inpatient & ICU & emergency & outpatient & Inpatient & ICU & emergency \\
\hline & $\begin{array}{l}(\mathrm{N}= \\
3061)\end{array}$ & $\begin{array}{l}(\mathrm{N}= \\
4390)\end{array}$ & $\begin{array}{l}(\mathrm{N}= \\
290)\end{array}$ & $(N=214)$ & $\begin{array}{l}(\mathrm{N}= \\
1730)\end{array}$ & $\begin{array}{l}(\mathrm{N}= \\
1413)\end{array}$ & $\begin{array}{l}(\mathrm{N}= \\
333)\end{array}$ & $(\mathrm{N}=618)$ \\
\hline Escherichia coli & $50 \%$ & $41 \%$ & $21 \%$ & $18 \%$ & $51 \%$ & $40 \%$ & $16 \%$ & $19 \%$ \\
\hline $\begin{array}{l}\text { Klebsiella } \\
\text { pneumoniae }\end{array}$ & $9 \%$ & $7 \%$ & $8 \%$ & $12 \%$ & $13 \%$ & $11 \%$ & $16 \%$ & $21 \%$ \\
\hline $\begin{array}{l}\text { Enterococcus } \\
\text { faecalis }\end{array}$ & $9 \%$ & $10 \%$ & $8 \%$ & $10 \%$ & $5 \%$ & $7 \%$ & $7 \%$ & $6 \%$ \\
\hline $\begin{array}{l}\text { Streptococcus } \\
\text { agalactiae }\end{array}$ & $6 \%$ & $3 \%$ & 0 & 0 & $2 \%$ & 0 & 0 & 0 \\
\hline $\begin{array}{l}\text { Pseudomonas } \\
\text { aeruginosa }\end{array}$ & $4 \%$ & $6 \%$ & $10 \%$ & $7 \%$ & $5 \%$ & $7 \%$ & $9 \%$ & $9 \%$ \\
\hline $\begin{array}{l}\text { Proteus } \\
\text { mirabilis }\end{array}$ & $3 \%$ & $3 \%$ & $4 \%$ & $4 \%$ & $4 \%$ & $5 \%$ & $8 \%$ & $8 \%$ \\
\hline $\begin{array}{l}\text { Staphylococcus } \\
\text { epidermidis }\end{array}$ & $3 \%$ & $3 \%$ & 0 & $2 \%$ & 0 & 0 & 0 & 0 \\
\hline $\begin{array}{l}\text { Enterococcus } \\
\text { faecium }\end{array}$ & $2 \%$ & $8 \%$ & $26 \%$ & $28 \%$ & $3 \%$ & $11 \%$ & $21 \%$ & $22 \%$ \\
\hline $\begin{array}{l}\text { Staphylococcus } \\
\text { aureus }\end{array}$ & $2 \%$ & $2 \%$ & $2 \%$ & $4 \%$ & 0 & 0 & 0 & $2 \%$ \\
\hline $\begin{array}{l}\text { Enterobacter } \\
\text { cloacae }\end{array}$ & 0 & $2 \%$ & 0 & 0 & 0 & $3 \%$ & 0 & 0 \\
\hline $\begin{array}{l}\text { Acinetobacter } \\
\text { baumannii }\end{array}$ & 0 & $2 \%$ & 0 & $2 \%$ & 0 & 0 & $6 \%$ & $3 \%$ \\
\hline $\begin{array}{l}\text { Klebsiella } \\
\text { oxytoca }\end{array}$ & 0 & 0 & 0 & 0 & $2 \%$ & 0 & 0 & 0 \\
\hline others (<2\%) & $12 \%$ & $13 \%$ & $21 \%$ & $13 \%$ & $15 \%$ & $16 \%$ & $17 \%$ & $10 \%$ \\
\hline
\end{tabular}

Escherichia coli, Klebsiella pneumoniae, Enterococcus faecalis, Enterococcus faecium, and Pseudomonas aeruginosa were the top 5 species in all age categories, and their antibiotic resistance rates sorted by different age categories were shown in Fig. 2-6 respectively. The resistance rates of cefperazone-sulbactam and piperacillin-tazobactam in Escherichia coli were low in all age categories, and the resistance rates of cephalosporins, carbapenems, and fluoroqinolones of Klebsiella pneumoniae were higher in geriatric overall. Enterococcus faecium was more resistant compared with Enterococcus faecalis in all age categories. Pseudomonas aeruginosa was mainly isolated from adult and geriatric, and the overall resistance rates were higher in adult except cefperazone-sulbactam (14.1\% vs. $24.2 \%, \mathrm{P}<$ 0.05). 


\section{Discussion}

UTIs are among the most common infections worldwide and caused predominantly by uropathogenic Escherichia coli (UPEC), which could lead to recurrence, renal damage, sepsis, or even death [6]. Thus early and appropriate empirical antibiotic therapy is of great importance. UTIs are a significant cause of morbidity in infant boys, older men, and females of all ages [1]. David et al. characterized the etiological profile of UTI in children < 2 years of age, showing that Escherichia coli proportion increased with age while Klebsiella spp. and Enterococcus spp. proportions decreased with age, with the average percentages of $56.9 \%, 14.1 \%$, and $11 \%$, respectively [14]. While the data of adults were different from children to some extent $[6,8,9]$. Thus the etiological profile and antibiotic susceptibility may change with age. However, few studies systematically compared the data of different ages, especially when subdivided by different ward types (outpatient, inpatient, ICU, or emergency) or gender (male or female). We presumed the underlying factors in these subgroups may be different, and age could be an important factor when considering empirical antibacterial therapy.

Although Escherichia coli in this study was still the dominant urinary pathogen in pediatric $(34 \%, \mathrm{n}=362)$, adult $(43 \%, \mathrm{n}$ $=3416)$, and geriatric $(44 \%, n=1617)$, the percentage was lower than the report from China mainland $(49.7 \%)$ [15], Taiwan (60.5\%) [16] and Ethiopia (52.7\%) [8]. One possible reason could be the proportion of complicated UTIs were higher than uncomplicated UTIs in our study, as both urology and nephrology departments in our hospital are the top 1 key discipline in China, with more intractable UTI patients visiting. The etiological profiles were also different in specific age populations, such as pediatrics [15], veterans [16], and pregnant women [17]. We also found the etiological profile was more diverse in adult than pediatric and geriatric patients, while the diversity was low in newborn. This is probably because the proportion of adult was the largest among all age categories, and the newborn were mostly from inpatients due to nosocomial or complicated UTIs. Furthermore, we analyzed the adult and geriatric patients subdivided by wards types. The proportion of Escherichia coli decreased, while some nosocomial infection associated pathogens (Klebsiella peumoniae, Pseudomonas aeruginosa, and Acinetobacter baumannii) increased in ICU and emergency department. More broad-spectrum antibiotics were used in ICU and emergency department compared with outpatient and non-ICU inpatient, which showed different etiological profile in these age categories in different ward types.

The dominant uropathogen in newborn was Eenterococcus faecium (45\%) instead of Escherichia coli (20\%). The proportion was lower than previous studies from West Asia and Europe (50\%-57\%) [18, 19], thus high proportion of nonE. coli uropathogens in newborns should be noticed when considering appropriate treatment. The percentage of Escherichia coli increased with age while the percentage of Enterococcus faecium decreased with age, which was in accordance with previous study [14]. The resistance rate of Enterococcus faecium was high to multiple available antibiotics in both newborn and pediatric, except vancomycin, linezolid, and teicoplanin. Markus et al. found that UTI caused by Enterococcus spp. was associated with male predominance, underlying urinary abnormality, and inappropriate empirical antibiotic therapy [20]. One possible explanation for the high percentage of Enterococcus faecium in newborn and pediatric of this study was that, a large proportion of them was from inpatient, and might be nosocomial infections. Thus more attention should be paid to urinary Enterococcus faecium in newborn and pediatric patients.

Antibiotic resistance is becoming a serious global health problem [21], and updated surveillance of antimicrobial susceptibility of specific type of infection is of great importance for initial empirical therapy [9]. The resistance rates of ampicillin, sulfamethoxazole-trimethoprim, and cephalosporins for Escherichia coli were higher in newborn and pediatric than in adult and geriatric overall, probably due to the inappropriate use of the these drugs, especially in the community setting. The resistance rates of carbapenems, pipercillin-tazobactam, and nitrofurantoin were $<5 \%$ in Escherichia coli overall, thus could be used for empirical treatment of UTI (Fig. 2). The resistance rate of Klebsiella pneumoniae was more serious compared with Escherichia coli (Fig. 3). The resistance rates of cephalosporins, fluoquinolones and carbapenems were higher in adult than in geriatric $(P<0.05)$. The overall resistance rates of Enterococcus faecium were 
higher than Enterococcus faecalis (Figs. 4 and 5, P<0.05). The resistance was more serious in adult and geriatric, with the trend of increasing with age.

This study has certain limitations: Firstly, due to its single-center and retrospective nature, the patients' selection bias existed and detailed clinical characteristics were not available. Secondly, although we enrolled a large sample size of 13308 non-repetitive patients from a tertiary general hospital, which has the top 1 discipline of nephrology and urology in China during a 12-year period, our data may not fully stand for the community or other specific population (e.g. mild or asymptomatic UTI patients), thus the conclusion should be extended with caution. Nevertheless, our results emphasized that the etiological profile and antibiotic susceptibility patterns of UTI patients were different among different age categories from large sample size, which are fundamental for designing proper antimicrobial therapy and provided the basis for high-quality research in the future.

\section{Conclusions}

The etiological profiles and antibiotic susceptibility patterns showed high divergence among different age categories in a tertiary general hospital during a 12-year period. Thus different antibiotic choices should be considered for treating patients of different ages.

\section{Declarations}

- Ethics approval and consent to partiticpate: The study was approved by the ethics committee of Peking University First Hospital (approval number: 2021-191).

- Consent for publication: We wish to submit the manuscript for publication in Annals of Clinical Microbiology and Antimicrobials, and the manuscript is not currently under consideration for publication in other journals.

- Availability of data and material: The datasets of current study are available from the corresponding author on reasonable request.

- Competing interests: The authors declare that they have no competing interests.

- Funding: This study was supported by the National Key Research and Development Program of China (2020YFC2005401).

- Authors' contribution: All the authors were active participants: $\mathrm{LH}$ and $\mathrm{CWH}$ were responsible for data collection, literature research, and manuscript writing; YY, LYS and HXL were responsible for data management, statistical analysis, and interpretation; LH was responsible for the design of the study, and revision of the paper. All authors read and approved the final manuscript.

- Acknowledgments: None.

\section{References}

1. Flores-Mireles AL, Walker JN, Caparon M, Hultgren SJ. Urinary tract infections: epidemiology, mechanisms of infection and treatment options. Nat Rev Microbiol. 2015;13(5):269-84.

2. Tullus K, Shaikh N. Urinary tract infections in children. Lancet. 2020;395(10237):1659-68.

3. Foxman B. Urinary tract infection syndromes: occurrence, recurrence, bacteriology, risk factors, and disease burden. Infect Dis Clin North Am. 2014;28(1):1-13.

4. Foxman B, Barlow R, D'Arcy H, Gillespie B, Sobel JD. Urinary tract infection: self-reported incidence and associated costs. Ann Epidemiol. 2000;10(8):509-15.

5. Hooton TM. Clinical practice. Uncomplicated urinary tract infection. N Engl J Med. 2012;366(11):1028-37. 
6. Klein RD, Hultgren SJ. Urinary tract infections: microbial pathogenesis, host-pathogen interactions and new treatment strategies. Nat Rev Microbiol. 2020;18(4):211-26.

7. Akram M, Shahid M, Khan AU. Etiology and antibiotic resistance patterns of community acquired urinary tract infections in JNMC Hospital Aligarh, India. Ann Clin Microbiol Antimicrob. 2007;6:4.

8. Bitew A, Molalign T, Chanie M. Species distribution and antibiotic susceptibility profile of bacterial uropathogens among patients complaining urinary tract infections. BMC Infect Dis. 2017;17(1):654.

9. China Antimicrobial Resistance Surveillance System. Antimicrobial resistance of bacteria from urine specimens: surveillance report from China Antimicrobial Resistance Surveillance System in 2014-2019. Chin J Infect Control. 2021;20(1):53-60.

10. Chu CM, Lowder JL. Diagnosis and treatment of urinary tract infections across age groups. Am J Obstet Gynecol. 2018;219(1):40-51.

11. Wilson ML, Gaido L. Laboratory diagnosis of urinary tract infections in adult patients. Clin Infect Dis. 2004;38(8):1150-8.

12. https://www.whonet.org.cn.

13. Clinical and Laboratory Standards Institute. Performance Standards for Antimicrobial Susceptibility Testing. CLSI document M100 31th edition. Wayne, Clinical and Laboratory Standard Institute; 2021.

14. Shaki D, Hodik G, Elamour S, Nassar R, Kristal E, Leibovitz R, et al. Urinary tract infections in children < 2 years of age hospitalized in a tertiary medical center in Southern Israel: epidemiologic, imaging, and microbiologic characteristics of first episode in life. Eur J Clin Microbiol Infect Dis. 2020;39(5):955-63.

15. Qiao LD, Chen S, Yang Y, Zhang K, Zheng B, Guo HF, et al. Characteristics of urinary tract infection pathogens and their in vitro susceptibility to antimicrobial agents in China: data from a multicenter study. BMJ Open. 2013;3(12):e004152.

16. DeRosa A, Carter MT, Wattengel BA, Lesse AJ, Sellick JA, Mergenhagen KA. Antimicrobial susceptibility trends for urinary isolates in the veteran population. Am J Infect Control. 2021;49(5):576-81.

17. Belete MA, Saravanan M. A systematic review on drug resistant urinary tract infection among pregnant women in developing countries in Africa and Asia; 2005-2016. Infect Drug Resist. 2020;13:1465-77.

18. Gurevich E, Tchernin D, Schreyber R, Muller R, Leibovitz E. Follow-up after infants younger than 2 months of age with urinary tract infection in Southern Israel: epidemiologic, microbiologic and disease recurrence characteristics. Braz J Infect Dis. 2016;20(1):19-25.

19. Alberici I, Bayazit AK, Drozdz D, Emre S, Fischbach M, Harambat J, et al. Pathogens causing urinary tract infections in infants: a European overview by the ESCAPE study group. Eur J Pediatr. 2015;174(6):783-90.

20. Giorgi LJ Jr, Bratslavsky G, Kogan BA. Febrile urinary tract infections in infants: renal ultrasound remains necessary. J Urol. 2005;173(2):568-70.

21. Boucher HW, Talbot GH, Bradley JS, Edwards JE, Gilbert D, Rice LB, et al. Bad bugs, no drugs: no ESKAPE! An update from the Infectious Diseases Society of America. Clin Infect Dis. 2009;48(1):1-12.

\section{Figures}




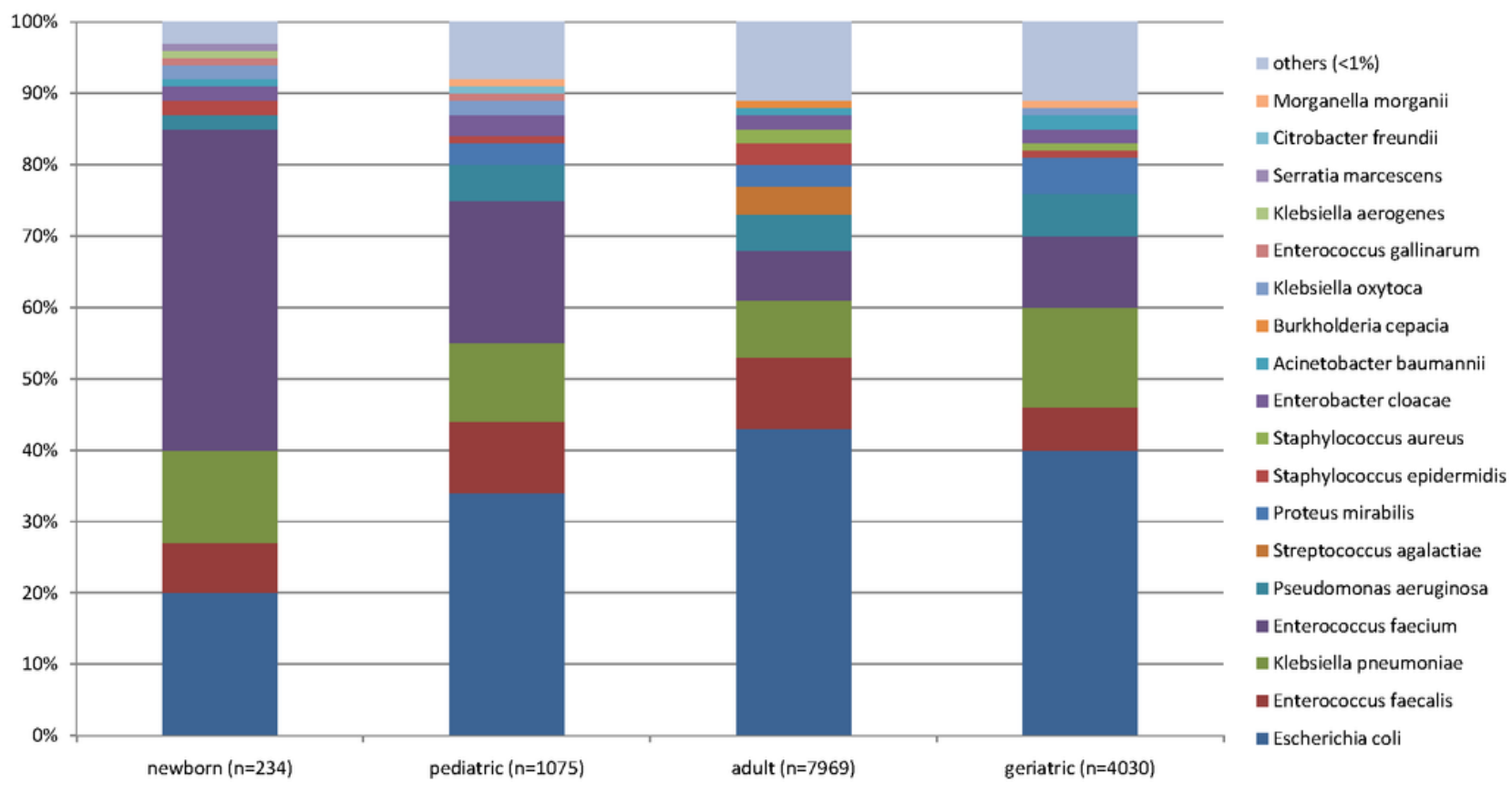

Figure 1

Distribution of urinary pathogens in different age categories. 


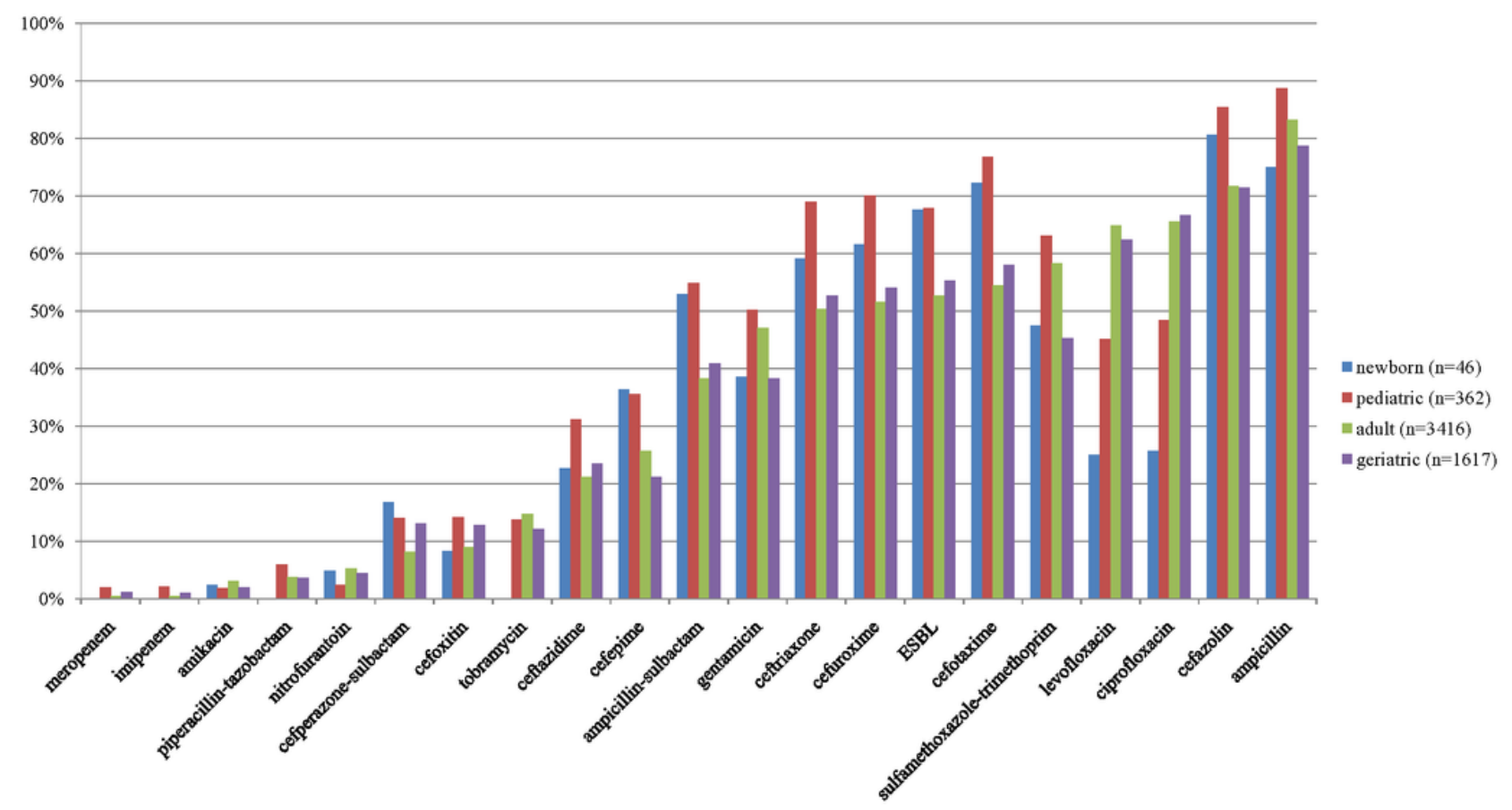

Figure 2

Antibacterial resistance rates of urinary Escherichia coli in different age categories. 


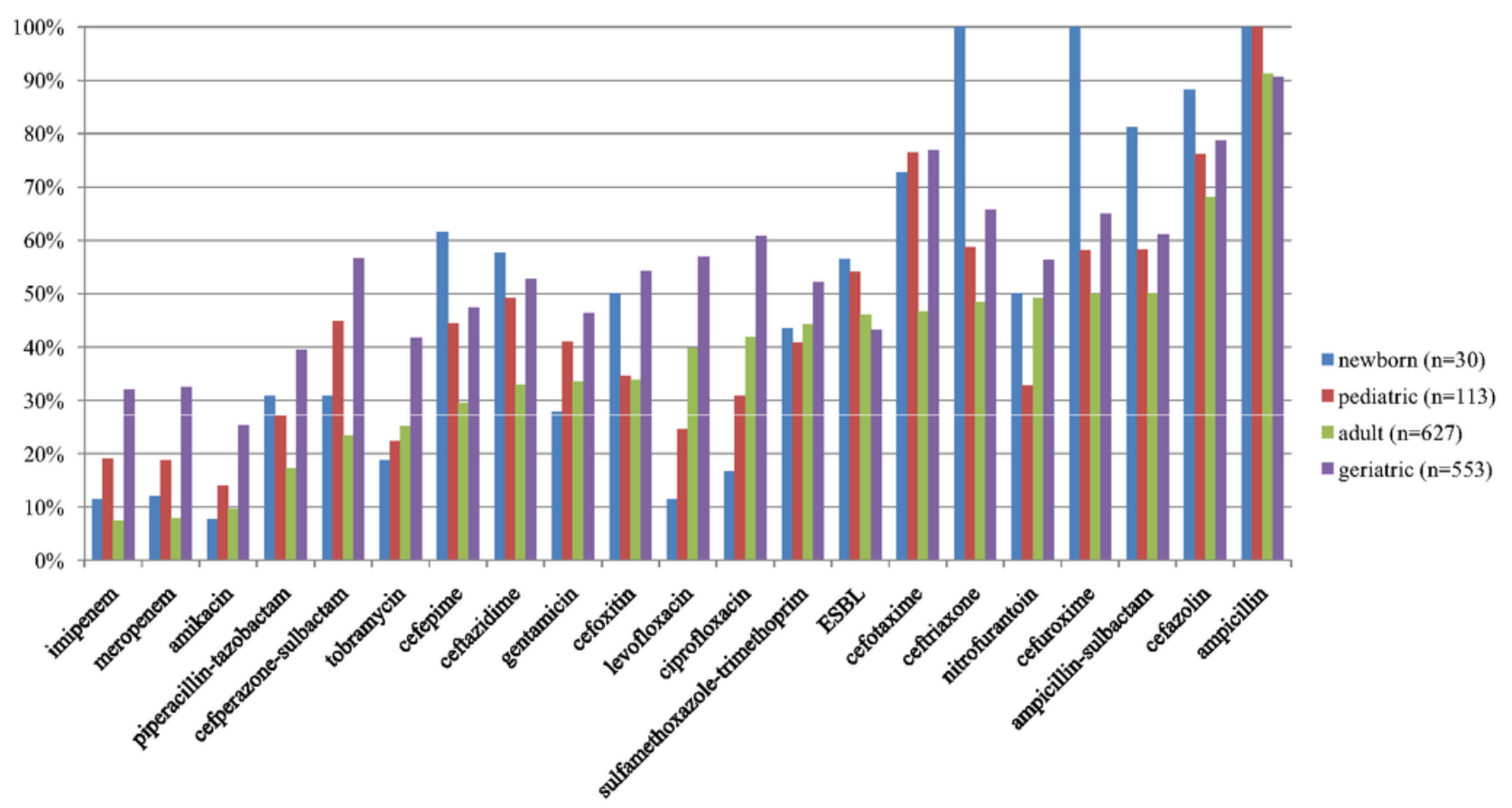

Figure 3

Antibacterial resistance rates of urinary Klebsiella pneumoniae in different age categories. 


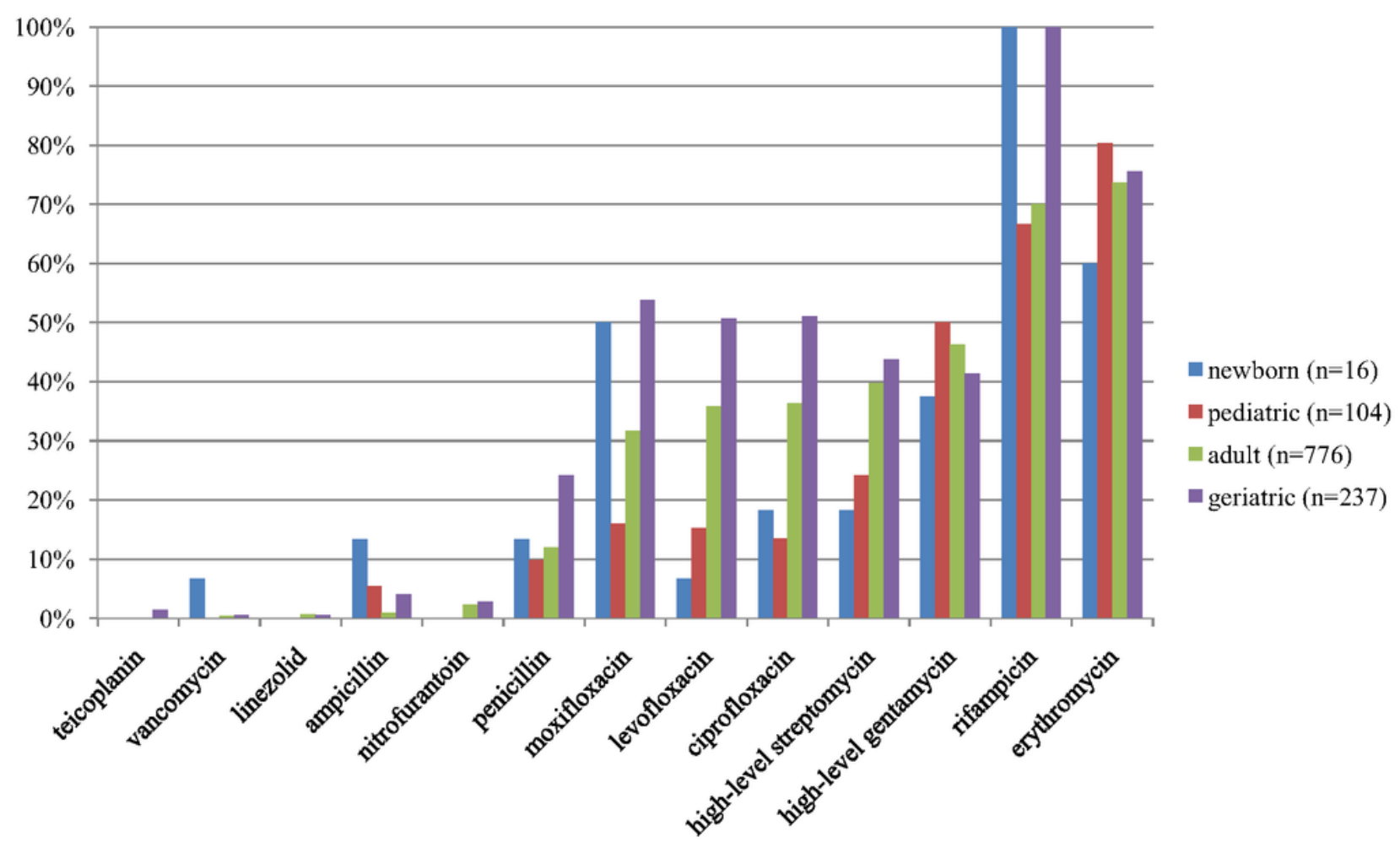

Figure 4

Antibacterial resistance rates of urinary Enterococcus faecalis in different age categories. 


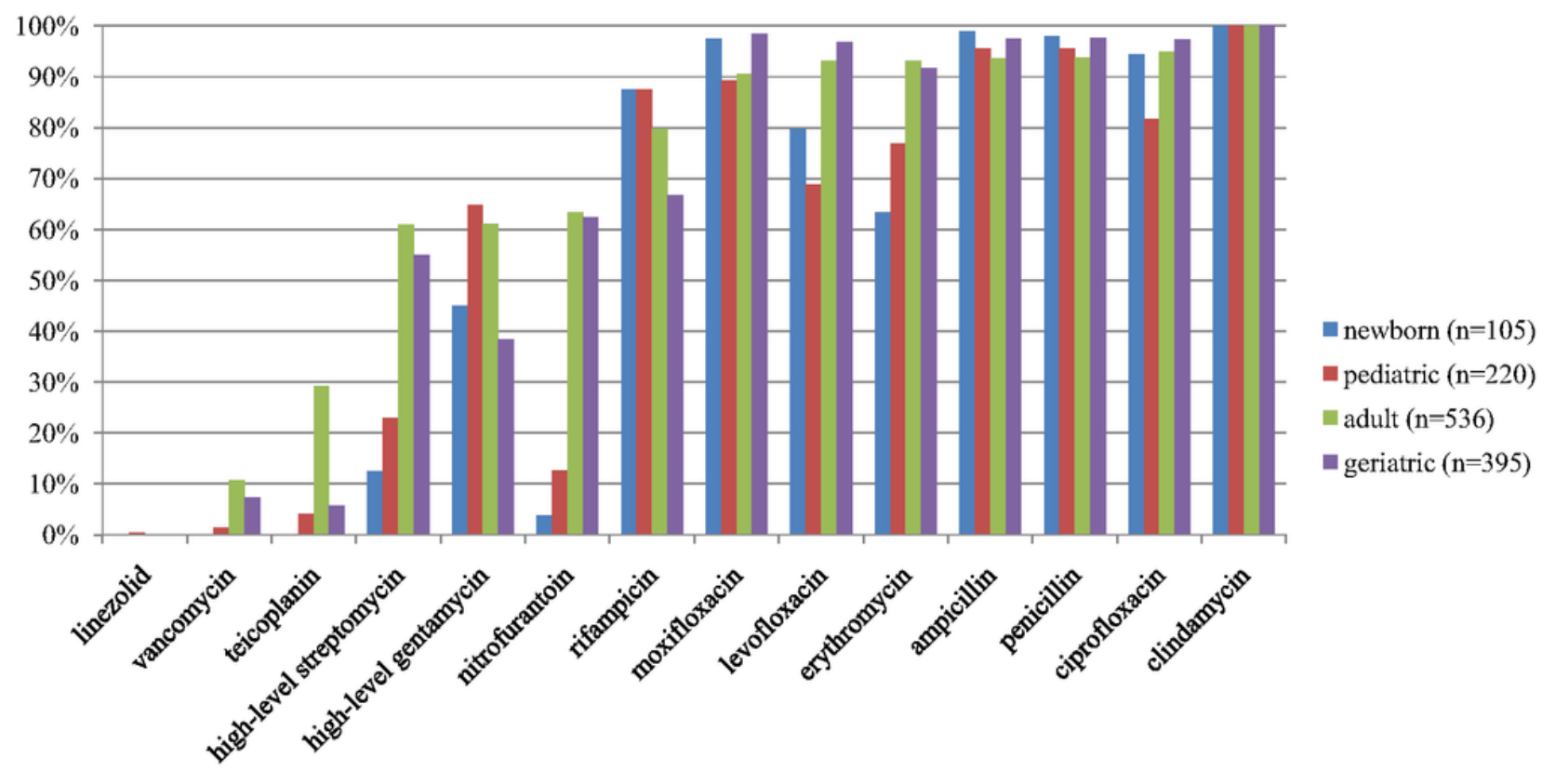

Figure 5

Antibacterial resistance rates of urinary Enterococcus faecium in different age categories. 


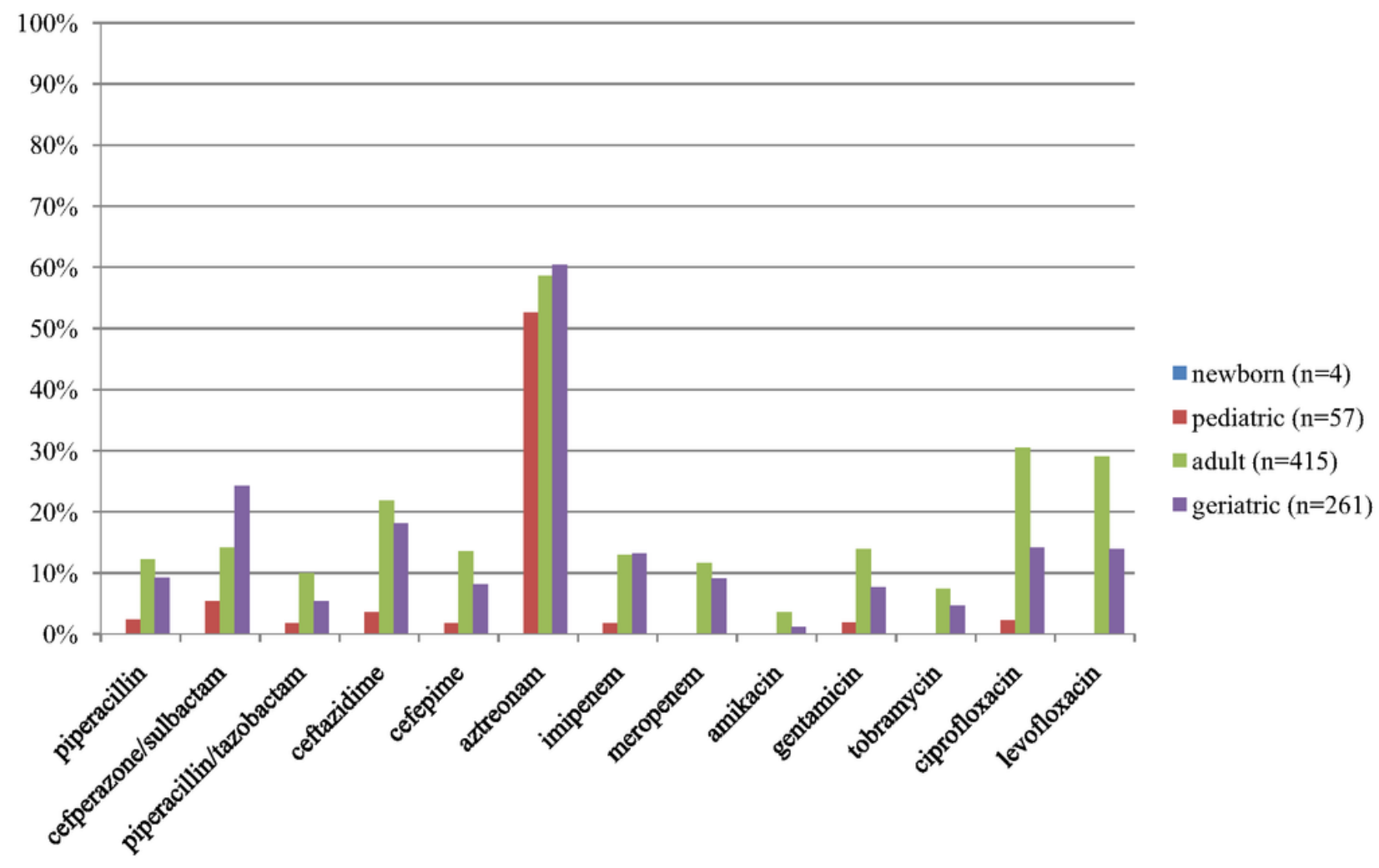

Figure 6

Antibacterial resistance rates of urinary Pseudomonas aeruginosa in different age categories. 